\section{OP0029 ASSOCIATION OF PLASMA MMP-3 LEVELS AND THE PRESENCE OF THE SHARED EPITOPE IN PATIENTS WITH RHEUMATOID ARTHRITIS}

S Drynda, C Kühne, J Kekow. Clinic of Rheumatology, Otto-Von-Guericke-University, Magdeburg, Germany

\subsection{6/annrheumdis-2001.921}

Background Rheumatoid arthritis (RA) is characterised by an overexpression of proinflammatory cytokines such as IL-1 and TNFa. These cytokines are known to stimulate the production of matrix degrading enzymes. The family of matrix metalloproteinases (MMP) are considered as key enzymes in the destruction of cartilage and bone. One of the most abundant MMP found in RA is MMP-3. It is well established that levels of MMP-3 in peripheral blood are highly correlated with disease activity in RA. Furthermore high plasma levels of MMP-3 account for an early erosive form of RA. It was the aim of our study to determine whether there exist an association between plasma MMP-3 levels and the presence of the shared epitope (SE) and an association of MMP-3 levels in plasma samples and bone extracts.

45 RA patients were included in this study. HLA-DRB genotyping with DNA from peripheral blood was performed using sequence-specific primers and reverse hybridisation provided by AID GmbH Strassberg. Plasma MMP-3 concentrations were determined applying the biotrak MMP-3 ELISA system by Amersham Pharmacia Biotech.

Our results show significant differences in plasma MMP-3 levels in RA patients depending on the presence of the shared epitope. The mean MMP-3 levels were significantly elevated in the SE positive compared to the SE negative group $(99.50+13.74$ $\mathrm{ng} / \mathrm{ml}$ vs. $43.02+9.23 \mathrm{ng} / \mathrm{ml}$, mean+SEM, $\mathrm{p}=0.012 \mathrm{Mann}$ Whitney U-test). The highest MMP-3 levels were found in patients with those allels encoding the amino acid sequence QRRAA at the position 70-74 in the third hypervariable region of the HLA-DRB1 molecule, i.e. "0101, "0102, "0404 and *0405. The MMP-3 levels in this group $(139.99+27.25 \mathrm{ng} / \mathrm{ml})$ were significantly higher compared not only to the SE negative group $(\mathrm{p}=0.011)$ but also compared to the group with the SE QKRAA $(68.17+6.53 \mathrm{ng} / \mathrm{ml}, \mathrm{p}=0.032)$.

Concentrations of MMP-3 in the extract of non-collagenous proteins from defatted bone powder were shown to be strongly correlated to levels in corresponding plasma samples $(r=0.57$, $\mathrm{p}<0.001$ ), suggesting that plasma MMP-3 levels reflect at least in part the local situation in bone.

Our data indicate that plasma MMP-3 levels in RA may reflect the situation in the bone and vary according to the presence of the shared epitope. This results may explain the association of early erosive RA with the presence of SE.

Objectives

Methods

Results

Conclusion
Background GlcN and GlcN-sulfate (GlcN-S) are being used extensively for the treatment of Osteoarthritis (OA). These compounds were thought to serve as nutritional substrates for the repair of damaged articular cartilage. However, controversy exists because free GlcN appears in the serum in very small amounts after ingestion of recommended doses for OA. Further, we had observed that GlcN added to Ch cultures, in concentrations similar to medium glucose, decreased $\mathrm{Ch}$ proliferation and PG synthesis. We now expand on these observations and report on the effects of $\mathrm{N}$-acylation and sulfation of the GlcN molecule on these biological effects.

Objectives To examine the effects of GlcN and related $\mathrm{N}$-acylated and sulfated analogues on $\mathrm{Ch}$ proliferation and PG synthesis.

Methods The compounds tested on isolated bovine, articular Ch, were: glucose (Glc), GlcN. HCl, N-acetyl GlcN (GlcNAc), $\mathrm{N}$-acetyl galactosamine (GalNAc), N-butyryl GlcN, (GlcNBu) GlcN-3,6-disulfate (GlcN-3,6-diS), GlcN-3.4.6- trisulfate (GlcN3,4,6-triS), adenosine triphosphate (ATP) or inosine. Following the various additions the $\mathrm{Ch}$ were harvested, at different time intervals, and enumerated or assessed for DNA synthesis by measuring $[3 \mathrm{H}]$-thymidine incorporation. For PG synthesis, confluent $\mathrm{Ch}$ cultures were treated with transforming growth factor (TFG)- b, [35S]-sulfate for 4 days. The media were analysed by Alcian blue and Toluidine blue precipitation, followed by gradient SDS-PAGE and autoradiography.

Results The addition of $\mathrm{GlcN}$. $\mathrm{HCl}(0.5-5.0 \mathrm{mM})$ to Ch cultures strongly inhibited cell proliferation, both in the absence and presence of TGF-b. This major antiproliferative effect of GlcN. $\mathrm{HCl}$ was reversed by GlcNAc, while GlcNBu resulted in a marked increase in $\mathrm{Ch}$ proliferation. Equivalent concentrations of $\mathrm{HCl}$ had no effect while sodium butyrate itself also showed antiproliferative effects. The addition of low $\mathrm{GlcN}$. $\mathrm{HCl}$ concentrations $(0.05-0.25 \mathrm{mM})$ resulted in minor stimulations of $\mathrm{Ch}$ proliferation and DNA synthesis. The antiproliferative effect of GlcN. $\mathrm{HCl}$ could not be reversed by the addition of ATP and inosine. Sulfation of GlcN in the 6-, 2-, and 2,6-positions, but not in the 3-, 2,3- and 3,6- positions reversed the GlcN. $\mathrm{HCl}$ effect. Sulfation at the 2- and 2,6-positions also reversed the $\mathrm{GlcN}$. $\mathrm{HCl}$ inhibition on DNA synthesis. Inosine $(2.5 \mathrm{mM})$ reversed the GlcN-3-S mediated antiproliferative effect on $\mathrm{Ch}$ at $8 \mathrm{~d}$ of treatment. The addition of GlcN also resulted in a dose dependent inhibition of the net PG synthesis over the range of 0.1-5.0 mM. N-acylation reversed, while sulfation partially reversed the $\mathrm{GlcN}$. $\mathrm{HCl}$ mediated PG inhibition. Inosine partially reversed the GlcN. HCl-dependent inhibition on PG synthesis.

Conclusion Free GlcN, in the form of $\mathrm{GlcN}$. $\mathrm{HCl}$, inhibited $\mathrm{Ch}$ proliferation and PG synthesis. These effects were apparently reversed by $\mathrm{N}$-acylation and, in a more complex pattern, by certain sulfation substitutions. N-butyryl GlcN, in particular, seems promising for further studies on the up-regulation of $\mathrm{Ch}$ growth and PG synthesis and in articular cartilage repair.

Available at Poster.

\section{THU0068 N-ACYLATION AND SULFATION OF GLUCOSAMINE (GLCN) DIFFERENTIALLY MODULATE CHONDROCYTE (CH) PROLIFERATION AND PROTEOGLYCAN (PG) SYNTHESIS}

T Anastassiades, D Terry, P Pezeshki, P Yang. Division of Rheumatology, Queen's University, Kingston, Canada 